\title{
State of charge estimation for lithium-ion batteries connected in series using two sigma point Kalman filters
}

\author{
Chi Nguyen Van ${ }^{1}$, Thuy Nguyen Vinh ${ }^{2}$ \\ ${ }^{1}$ Institute of High-Technology Research and Development for Industry (RIAT), Thai Nguyen University of Technology, Thai Nguyen, \\ Vietnam \\ ${ }^{2}$ Department of Automation, Thai Nguyen University of Technology (TNUT), Thai Nguyen, Vietnam
}

\begin{abstract}
Article Info
Article history:

Received Apr 19, 2021

Revised Aug 3, 2021

Accepted Sep 1, 2021

Keywords:

Battery pack

Lithium-ion battery

Sigma point Kalman filter

SoC estimation

State of charge

ABSTRACT

This paper proposes a method to estimate state of charge (SoC) for Lithiumion battery pack (LIB) with $N$ series-connected cells. The cell's model is represented by a second-order equivalent circuit model taking into account the measurement disturbances and the current sensor bias. By using two sigma point Kalman filters (SPKF), the SoC of cells in the pack is calculated by the sum of the pack's average SoC estimated by the first SPKF and SoC differences estimated by the second SPKF. The advantage of this method is the SoC estimation algorithm performed only two times instead of $N$ times in each sampling time interval, so the computational burden is reduced. The test of the proposed SoC estimation algorithm for 7 samsung ICR18650 Lithium-ion battery cells connected in series is implemented in the continuous charge and discharge scenario in one hour time. The estimated SoCs of the cells in the pack are quite accurate, the 3-sigma criterion of estimated SoC error distributions is $0.5 \%$.
\end{abstract}

This is an open access article under the CC BY-SA license.

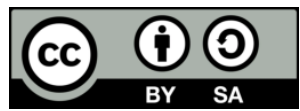

\section{Corresponding Author:}

Chi Nguyen Van

Institute of High-Technology Research and Development for Industry (RIAT), Thai Nguyen University of Technology National 666, 3/2 Street, Tich Luong Ward, Thai Nguyen City 251750, Vietnam

Email: ngchi@tnut.edu.vn

\section{INTRODUCTION}

Lithium-ion batteries pack (LIB) are so far known to be the most commonly used batteries with the following advantages: a higher energy density, voltage capacity; lower self-discharge rate than other rechargeable batteries; the price is much cheaper than the LiPo battery. LIBs power almost every portable devices such as smartphone, laptop, and tablet sold today across the world. Now, LIBs are applied to not only electric cars, electric motorcycles, buses, trucks but also large-scale electrical energy storage. LIB is formed by many cells connected in series and parallel in order to get the desired voltage and power [1] as shown in Figure 1. The largest market for LIBs has traditionally been portable electronic devices, but in the near future there is an extensive growth in the demand for LIBs in transportation and electrical energy storages [2]. The state of charge (SoC) is an important input for balancing, energy and power calculations of LIB. SoC depends on the open-circuit-voltage (OCV) with the nonlinear relationship affected by the temperature, aging, and charge/discharge mode, [3]-[5]. The OCV depends on temperature and electrode particle surface concentration, but $\mathrm{SoC}$ depend on particle average concentration. In the practice, optimal safe operation range of $\mathrm{SoC}$ for cells is above $20 \%$ and below $90 \%$. Figure 2 describes a typical relationship between SoC and $\mathrm{OCV}$ at $-5{ }^{\circ} \mathrm{C}$ and $25^{\circ} \mathrm{C}$ of temperature for a Lithium samsung cell. SoC is a parameter that is not measurable directly, so it is estimated by using the current, voltage, and temperature of the cell. SoC is needed to be estimated accurately in order to use for the SoC balance control problem. Controlling the 
operation of the battery system in order to prolong the life of the battery cells, to operate safely is a topic that scientists are currently interested in applying LIBs in transportation and electrical energy storage. To estimate SoC for one cell, we need the model describing the dynamic behavior of the cell and the SoC estimation algorithm based on that model with real-time current, voltage, and temperature inputs. The accuracy of the estimated SoC depends on the model of the cell and the SoC estimation algorithms used [6].

The model of the cell: if the complex model is used (such as an electrochemical model), the estimated SoCs will be more accurate, but the computational burden and hardware costs will increase a lot when the number of cells in series $N$ is large. If the equivalent-circuit model is used, the order of that model plays an important role. The computational complexity of the SoC estimation algorithm is simpler with the lower-order model but the cell dynamic behavior is not reflected accurately. There is higher accuracy in SoC estimation when the higher-order model is used, but the computational burden is increased [7]. The SoC estimation algorithm: Up to now, most of the SoC estimation algorithms are based on coulomb counting [8], Kalman filter (KF) [9], adaptive extended Kalman filter (EKF) [10], [11], and other [12], [13] improved algorithms. Several other algorithms such as the use of fuzzy models and neural networks are also applied [14], [15].

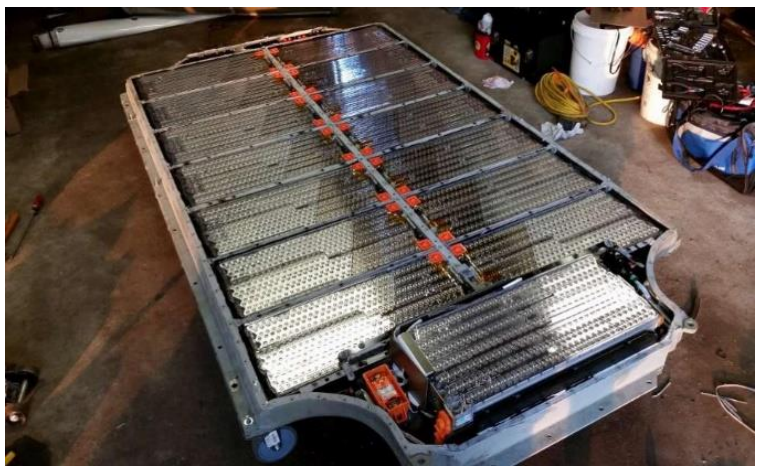

Figure 1. Inside the tesla model S battery pack

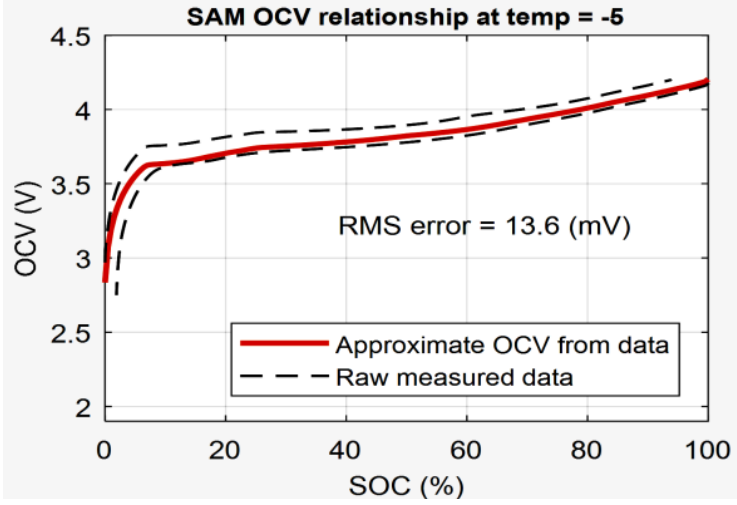

(a)

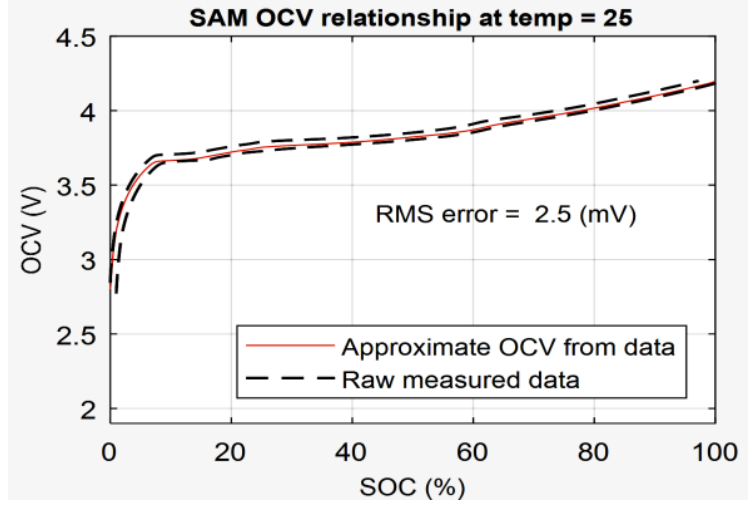

(b)

Figure 2. The typical relationship between SoC and OCV for a lithium samsung cell: (a) tested at $-5^{\circ} \mathrm{C}$ and (b) tested at $25{ }^{\circ} \mathrm{C}$

The SoC estimation for $N$ cell connected in series is the challenge problem, there are some works related to this in literature [16], [17]. Normally, the SoC estimation algorithm is implemented repeatedly $N$ times, if the number of cells $N$ is large then this is really a computational burden for the battery management system [18]. Beside that, from the works [7], [12], [14], [17] and the actual testing we found that the interference of the measuring noises, current sensor bias are an obstacle to estimating SoC exactly. In practice, the current through the cell is usually measured by a shunt resistor with a voltage signal of $m V$, this voltage signal is more susceptible to interference than the voltage measuring signal at both ends of the cell $(2.7 \mathrm{~V}$ to $4.2 \mathrm{~V})$. The current sensor bias of the rectifier affected by temperature needs to be considered in the 
current measurement. The KF theory assumes that all noises are zero mean, so an unknown current sensor bias can introduce permanent SoC error. This paper introduces the SoC estimation method for a $\mathrm{LiB}$ connected in series using sigma point Kalman filters (SPKF) to minimize the influence of current and vfoltage measurement noise on the estimated SoC results. By using the second-order equivalent circuit model (SECM), this allows to describe more exactly the cell's dynamic in the charged and discharged scenarios with amplitude varying suddenly (an important feature of electrical vehicles using LIBs must be considered) [19]. This method uses two SPKFs, the SoC of cells in the pack is calculated by sum of pack's average SoC estimated by first SPKF and SoC differences estimated by second SPKF.

The contributions of this work are: The first, by using only two filters to estimate the $N$ SoCs of $N$ cells connected in series, the SoC estimation algorithm is performed only two times instead of $N$ times in each sampling time interval, so the computational burden reduced to $N-2$ times, this is a very effective solution when the battery pack has a large number of cells. The second, the SoC estimation taking into account the current sensor bias in the model in association with applying the SPKF filter allows mapping the distributions of current, voltage, and temperature signals to the distribution of the estimated SoCs of the cells with more accuracy tested by the $3 \sigma$ criterion. The remaining part of this paper is structured as follows: section 2 mentions the SECM of the cell taking into account the measurement noises and the current sensor bias. Section 3 introduces the proposed SoC estimation algorithm of cells connected in series using 02 SPKFs. Section 4 shows the results applied on the Lithium ICR18650-22P SAMSUNG cells and discussions. Finally, section 5 summarizes some conclusions.

\section{THE SECOND-ORDER EQUIVALENT CIRCUIT MODEL OF CELL}

Suppose that the LIB's cell is depicted as in Figure 3(a), in which $i(t)$ is the cell current $(i(t)>0$ for charge and $i(t)<0$ for discharge, $i(t)=0$ for cell in no charge/discharge), $10 \%<z(t)<95 \%$ is SoC, $3.6 \mathrm{~V}<v(t)<4.2 \mathrm{~V}$ is the cell voltage, $-5^{0} \mathrm{C}<T(t)<50^{\circ} \mathrm{C}$ is cell temperature. In order to estimate the SoC at the sample time $k$, which is $Z_{k}$, we need to measure the voltage, current and cell temperature. Let $i_{k}+b_{k}+n_{i, k}$ is the measured current, $v_{k}+n_{v, k}$ is measured voltage, in which $b_{k}$ is the current sensor bias; $n_{i, k}$ is the current noise; $n_{v, k}$ is the voltage noise. The Figures 3(b), 3(c) are the recorded voltage and current in the practice, respectively. The scanning electrochemical microscopy (SECM) of the cell is plotted in Figure 4 with $\operatorname{OCV}(z(t), T)$ is the relationship between the oral cholera vaccine (OCV) and SoC, this relationship is nonlinear function depending on cell temperature and this function is needed to determine by the practice data collected by tests. The parameter $h(t)$ is the dynamic hysteresis voltage as a function of SoC and time, $R_{1}(T), C_{1}(T), R_{2}(T), C_{2}(T)$ are two resistor-capacitor pairs describe the cell dynamic, $R_{0}(T)$ is series resistance parameter, $M_{0}(T), M(T)$ are the hysteresis parameters, $\eta(T)$ is Coulombic efficiency, $\gamma(T)$ is the parameter related to the the rate of decay. Note that all parameters of cell depend on the cell temperature. In exact terms, they also depend on the cell aging, the charge/discharge condition. For different kind of cell (Samsung, Panasonic), they are different. In this work, we suppose that all parameters of cell only depend on the cell temperature.

Suppose that all the quantities is discreterized at the sampling time $k, k=0,1,2, \ldots, \infty$ with the sampling interval $\Delta t$ is small enough so that the current and voltage of cell are constant over that interval. Suppose that $Q$ is total capacity of a cell, the change in SoC over sampling interval is (1).

$$
z_{k+1}=z_{k}-i_{k} \frac{\eta_{k} \Delta t}{Q}
$$

The diffused resistor currents are computed as (2),

$$
\begin{aligned}
& i_{R_{1}, k+1}=\exp \left(-\frac{\Delta t}{R_{1} C_{1}}\right) i_{R_{1}, k}+\left(1-\exp \left(-\frac{\Delta t}{R_{1} C_{1}}\right)\right) i_{k} \\
& i_{R_{2}, k+1}=\exp \left(-\frac{\Delta t}{R_{2} C_{2}}\right) i_{R_{2}, k}+\left(1-\exp \left(-\frac{\Delta t}{R_{2} C_{2}}\right)\right) i_{k}
\end{aligned}
$$

Hysteresis voltage is given by (3).

$$
h_{k+1}=\exp \left(-\left|\frac{i_{k} \eta_{k} \Delta t}{Q}\right|\right) h_{k}-\left(1-\exp \left(\left|\frac{i_{k} \eta_{k} \Delta t}{Q}\right|\right)\right) \operatorname{sign}\left(i_{k}\right)
$$




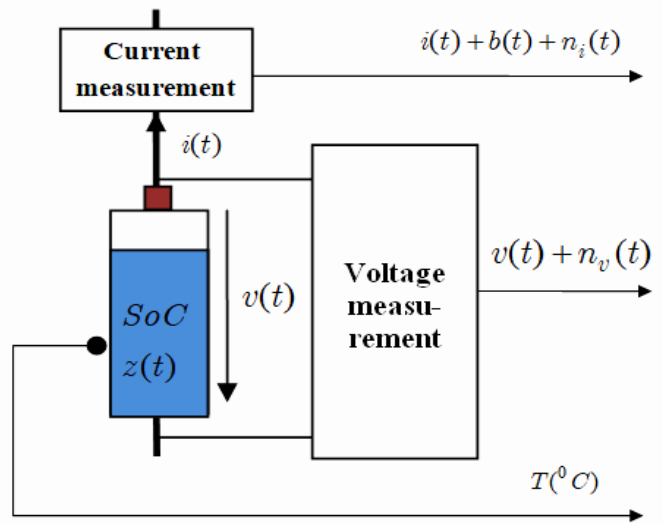

(a)

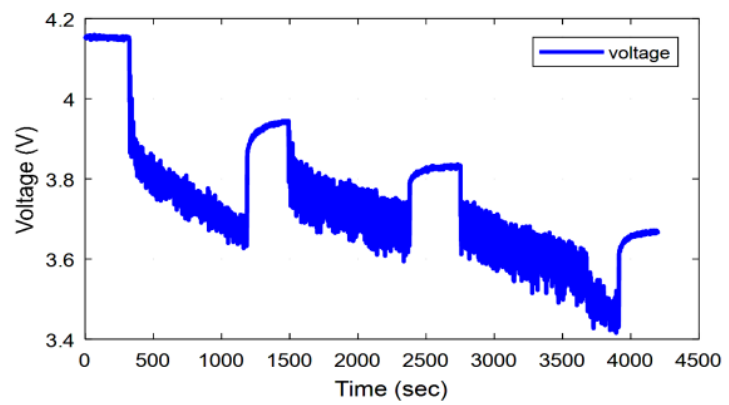

(b)

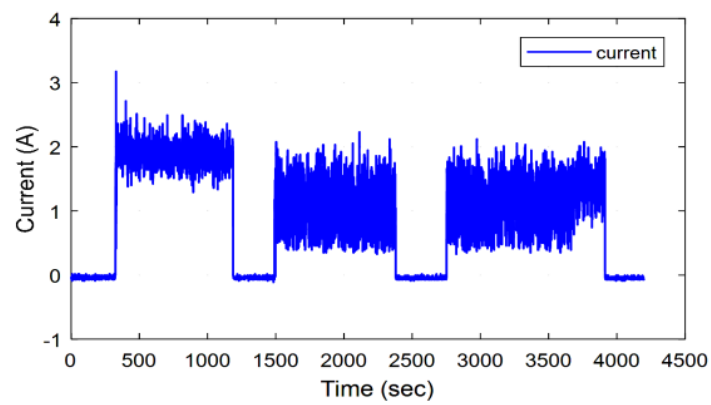

(c)

Figure 3. Illustration of the cell measurements, (a) the LIB, (b) the recorded voltage (c) current of cell

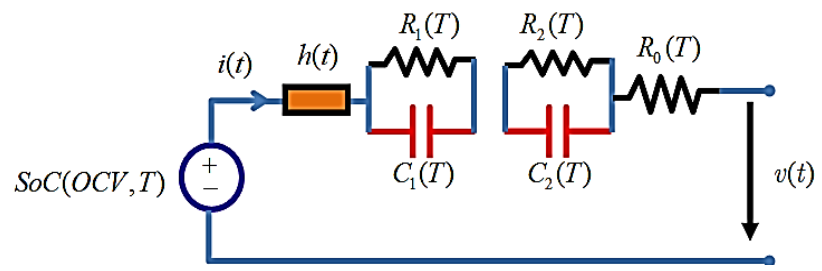

Figure 4. The second-order equivalent circuit model of the cell

The current sensor bias is $b_{k}=b_{k-1}+n_{k-1}$, in which $n_{k-1}$ is virtual noise of the current sensor bias used only for the calculation. The output equation of the model describing the relationship of the cell voltage, the current, SoC, the hysteresis, and the diffused resistor currents is written as (4).

$$
y_{k}=\operatorname{OCV}\left(z_{k}\right)+M h_{k}-R_{1} i_{R_{1}, k}-R_{2} i_{R_{2}, k}-R_{2}\left(i_{k}-b_{k}\right)
$$

The state vector related to the diffused resistor currents, the hysteresis, SoC and the current sensor bias as is formed as the (5).

$$
\mathrm{x}_{k}=\left[\begin{array}{lllll}
i_{R_{1}, k+1} & i_{R_{2}, k+1} & h_{k+1} & z_{k+1} & b_{k+1}
\end{array}\right]^{T}
$$

Define the system matrices and output matrix of the model as.

$$
\mathrm{A}_{R C}=\left[\begin{array}{cc}
\exp \left(-\frac{\Delta t}{R_{1} C_{1}}\right) & 0 \\
0 & \exp \left(-\frac{\Delta t}{R_{2} C_{2}}\right)
\end{array}\right], \mathrm{B}_{R C}=\left[\begin{array}{c}
1-\exp \left(-\frac{\Delta t}{R_{1} C_{1}}\right) \\
1-\exp \left(-\frac{\Delta t}{R_{2} C_{2}}\right)
\end{array}\right], \mathrm{A}_{H, k}=\exp \left(\left|\frac{i_{k} \eta_{k} \Delta t}{Q}\right|\right)
$$




$$
\mathrm{A}\left(i_{k}\right)=\left[\begin{array}{ccc}
1 & 0 & 0 \\
0 & \mathrm{~A}_{R C} & 0 \\
0 & 0 & \mathrm{~A}_{H, k}
\end{array}\right], \mathrm{B}\left(i_{k}\right)=\left[\begin{array}{cc}
-\frac{\eta_{k} \Delta t}{Q} & 0 \\
\mathrm{~B}_{R C} & 0 \\
0 & 1-\mathrm{A}_{H, k}
\end{array}\right], \mathrm{C}_{k}=\left[\begin{array}{llll}
0 & -R_{1} & -R_{2} & M
\end{array}\right], \mathrm{D}_{k}=-R_{0}
$$

Suppose the input and output vectors of the model as (6).

$$
\mathrm{u}_{k}=\left[\begin{array}{c}
i_{k}+b_{k} \\
\operatorname{sign}\left(i_{k}+b_{k}\right)
\end{array}\right], y_{k}=v_{k}
$$

Finally, the SECM of the cell is written as (7).

$$
\left\{\begin{array}{c}
\mathrm{x}_{k+1}=\mathrm{A}\left(i_{k}\right) \mathrm{x}_{k}+\mathrm{B}\left(i_{k}\right) \mathrm{u}_{k} \\
y_{k}=\operatorname{OCV}\left(z_{k}, T_{k}\right)+\mathrm{Cx}_{k}+\mathrm{Du}_{k}
\end{array}\right.
$$

Model (7) is used to estimate the SoC for the cell with input $\mathrm{u}_{k}$ and output $y_{k}$ without measurement noise. When measuring $\mathrm{u}_{k}$ and $y_{k}$, the two measurement noises $n_{i, k}$ and $n_{v, k}$ were mixed in the curent and voltage as described in Figure 3(a). So that, the input vector and output vector of the model is written as (8).

$$
\mathrm{u}_{n, k}=\left[\begin{array}{c}
i_{k}+b_{k}+n_{i, k} \\
\operatorname{sign}\left(i_{k}+b_{k}+n_{i, k}\right)
\end{array}\right], y_{n, k}=v_{k}+n_{v, k}
$$

With the input and output vectors in the (8), the model (7) is rewritten as (9).

$$
\left\{\begin{array}{c}
\mathrm{x}_{n, k+1}=\mathrm{A}\left(i_{n, k}\right) \mathrm{x}_{n, k}+\mathrm{B}\left(i_{n, k}\right) \mathrm{u}_{n, k} \\
y_{n, k}=\operatorname{OCV}\left(z_{n, k}, T_{k}\right)+\mathrm{Cx}_{n, k}+\mathrm{Du}_{n, k}
\end{array}\right.
$$

In which $\mathrm{x}_{n, k+1}$ is state vector affected by measurement noise formed as (10).

$$
\mathrm{x}_{n, k}=\left[\begin{array}{lllll}
i_{R_{1} n, k+1} & i_{R_{2} n, k+1} & h_{n, k+1} & z_{n, k+1} & b_{n, k+1}
\end{array}\right]^{T}
$$

Suppose the error $\mathrm{w}_{k}=\mathrm{x}_{k+1} \mathrm{x}_{n, k+1}$ to be the system noise of the model, $\xi_{k}=y_{k}-y_{n, k}$ is the output noise of the model, we have (11).

$$
\left\{\begin{array}{l}
\mathrm{x}_{k+1}=\mathrm{A}\left(i_{n, k}\right) \mathrm{x}_{n, k}+\mathrm{B}\left(i_{n, k}\right) \mathrm{u}_{n, k}+\mathrm{w}_{k} \\
y_{k}=\operatorname{OCV}\left(z_{k}, T_{k}\right)+\mathrm{Cx}_{k}+\mathrm{Du}_{n, k}+\xi_{k}
\end{array}\right.
$$

The model (11) is used to estimate the state vector $\mathrm{x}_{k+1}$, in that vector there are the SoC $z_{k}$ and the current sensor bias $b_{k}$ need to be estimated. In this work, we use LIB Samsung ICR18650-22P [20] with parameters $Q=2200 \mathrm{mAh}, V_{\max }=4.25 \mathrm{~V}, V_{\min }=2.75 \mathrm{~V}$, nominal voltage $3.62 \mathrm{~V}$. The test temperature range is considered from $-5{ }^{\circ} \mathrm{C}$ to $45^{\circ} \mathrm{C}$. The values of model's parameters determined by practical tests are given in Table 1.

Table 1. The cell's model parameters of LIB Samsung ICR18650-22P

\begin{tabular}{ccccccc}
\hline $\mathrm{T}^{0} \mathrm{C}$ & $-5{ }^{0} \mathrm{C}$ & $5{ }^{0} \mathrm{C}$ & $15^{0} \mathrm{C}$ & $25{ }^{0} \mathrm{C}$ & $35{ }^{0} \mathrm{C}$ & $45{ }^{0} \mathrm{C}$ \\
\hline$\eta_{\mathbf{k}}$ & 1.0869 & 0.9803 & 1.0220 & 0.95 & 1.0542 & 1.0399 \\
$\mathbf{Q}(\mathbf{A h})$ & 2.1596 & 2.1877 & 2.1943 & 2.175 & 2.1515 & 2.1523 \\
$\gamma$ & 243.1946 & 78.4915 & 63.6762 & 250.0074 & 170.6407 & 151.3064 \\
$\mathbf{M}_{\mathbf{0}}(\mathbf{V})$ & 0.0072 & 0.0049 & 0.0035 & 0.002 & 0.0036 & 0.0024 \\
$\mathbf{M}(\mathbf{V})$ & 0.0347 & 0.0257 & 0.0218 & 0.02 & 0.0201 & 0.0185 \\
$\mathbf{R}_{\mathbf{0}}(\Omega)$ & 0.013 & 0.023 & 0.0292 & 0.033 & 0.0012 & 0.0011 \\
$\mathbf{R}_{\mathbf{1}} \mathbf{C}_{\mathbf{1}}(\mathbf{s})$ & 0.6124 & 1.7555 & 0.3227 & 0.899 & 0.6997 & 0.4630 \\
$\mathbf{R}_{\mathbf{2}} \mathbf{C}_{\mathbf{2}}(\mathbf{s})$ & 3.9035 & 7.5994 & 8.1118 & 10.023 & 8.1840 & 6.5319 \\
$\mathbf{R}_{\mathbf{1}}(\Omega)$ & 0.0204 & 0.0203 & 0.0201 & 0.019 & 0.019 & 0.0019 \\
$\mathbf{R}_{\mathbf{2}}(\Omega)$ & 0.0494 & 0.0376 & 0.0288 & 0.022 & 0.0136 & 0.0134 \\
\hline
\end{tabular}




\section{PROPOSED SOC ESTIMATION ALGORITHM FOR $N$ CELLS CONNECTED IN SERIES USING TWO SPKFS}

Suppose that there is $N$ cells LIB connected in series as shown in Figure 5, the problem is to estimate SoCs of $N$ cells with the input and output signals affected by noises as (8), the noise distributions are considered as Gaussian-distributed. SPKF is the filter with high precision aimed at nonlinear system, in practice, the SPKF has some advantages compared to the EKF. This filter allows performance KF for nonlinear systems without the linearization steps. The set of weighted samples, called sigma points, is used for normalizing mean and covariance of a probability distribution. The sigma points of input distributions are mapped thought the nonlinear dynamic model functions to get the sigma points of output distribution [21], [22]. SPKF allows estimation states of nonlinear dynamic systems more accurately than the EKF does, but the computational complexity is same as EKF [23]-[25]. SPKF is easily implemented because there is no requirement of the Jacobi matrix calculation. The accuracy of state estimation using the SPKF mainly depends on the strategies of choosing sigma-points [26], [27]. Normally, we need to estimated SoC $N$ times for $N$ cells. If the number of cells $N$ is large, there is a large amount of calculation [18], [19]. To reduce the SoC estimation times, in this work we use only two SPKFs, the first filter estimates the mean of $N$ SoC and the current sensor bias, the second filter estimates $N$ SoC differences from the average SoC, the estimated $\mathrm{SoC}$ of each cell then is calculated by the sum of the average SoC determined by the first filter and the difference SoC estimated by the second filter. In one sampling time interval, the SoC estimation algorithm is implemented two times instead of $N$ times.

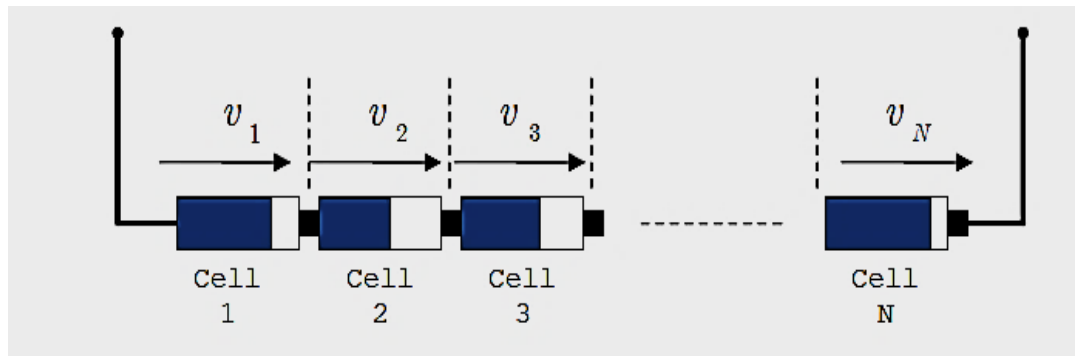

Figure 5. $N$ LIB cells connected in series

Consider the second model of cell in the (11) with input and output in the (8). The SOC estimation algorithm flowchart for $N$ cell connected in series is conducted as:

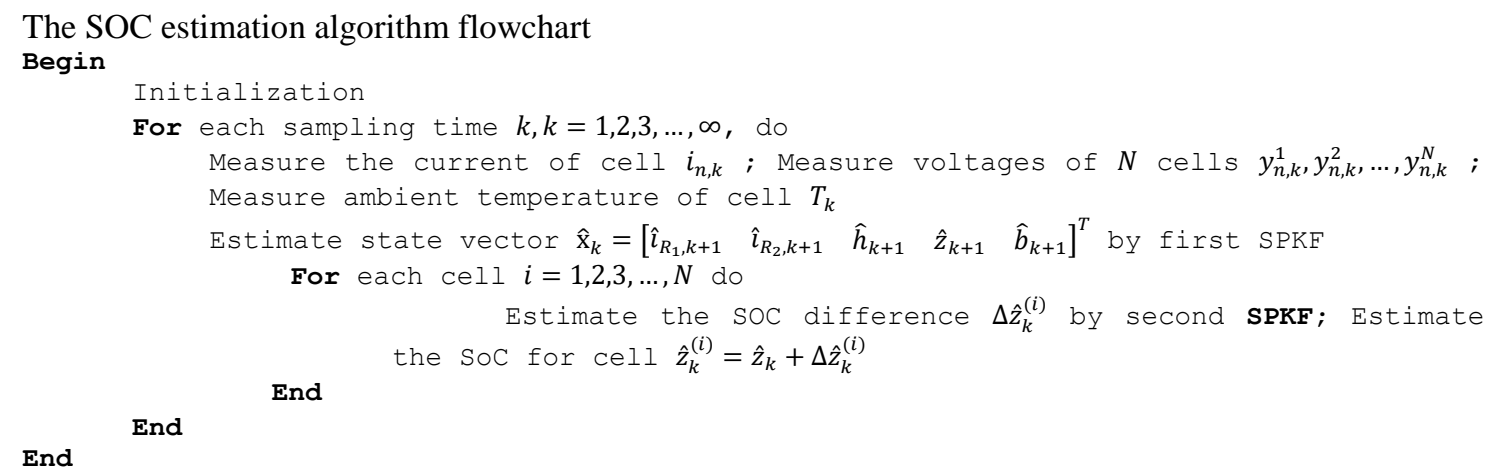

\subsection{Algorithm to estimate the average SoC for the $\mathrm{N}$ cells by the first SPKF filter}

The first filter estimates the mean of $N$ SoC and the current sensor bias as steps following:

- Form the augmented state vector at sampling time $k$ defined by $\hat{\mathrm{x}}_{k-1}^{a+}$, including the estimated state vector at the sampling time $k-1$ the mean vector of the system noise $\overline{\mathrm{w}}$ and mesurement noise $\bar{\xi}$ :

$$
\hat{\mathrm{x}}_{k-1}^{a,+}=\left[\begin{array}{lll}
\hat{\mathrm{x}}_{k-1}^{+} & \overline{\mathrm{W}} & \bar{\xi}
\end{array}\right]
$$

- Form the augmented covariance matrix (CM) of state estimation error vector by using $\mathrm{CM}$ of state estimation error at sampling time $k-1$, the $\mathrm{CM}$ of system noises and the $\mathrm{CM}$ of measurement noises: 


$$
\sigma_{\tilde{\mathrm{x}}, k-1}^{a,+}=\operatorname{diag}\left[\sigma_{\tilde{\mathrm{x}}, k-1}^{+} \quad \sigma_{\mathrm{w}} \quad \sigma_{\xi}\right]
$$

- Set up matrix of $p+1$ sigma points:

$$
\mathrm{X}_{k-1}^{a,+}=\left\{\hat{\mathrm{x}}_{k-1}^{a,+}, \hat{\mathrm{x}}_{k-1}^{a,+}+\gamma \sqrt{\sigma_{\tilde{\mathrm{x}}, k-1}^{a,+}}, \hat{\mathrm{x}}_{k-1}^{a,+}-\gamma \sqrt{\sigma_{\tilde{\mathrm{x}}, k-1}^{a,+}}\right\} \triangleq\left[\begin{array}{lll}
\mathrm{X}_{k-1}^{\mathrm{X},+} & \mathrm{X}_{k-1}^{w,+} & \mathrm{X}_{k-1}^{\xi,+}
\end{array}\right]^{T}
$$

- $\quad$ Calculate sigma points matrix at sampling time $k$ by using the cell model's dynamic function in (11):

$$
\mathrm{X}_{k, j}^{\mathrm{X},-}=\mathrm{A}_{k}\left(i_{n, k}\right) \mathrm{X}_{k-1, j}^{\mathrm{X},+}+\mathrm{B}_{k}\left(i_{n, k}\right) \mathrm{u}_{n, k}+\mathrm{X}_{k-1, j}^{w,+} \quad j=0,1,2, \ldots p
$$

- Estimate the priori state vector at the sampling time $k$ :

$$
\hat{\mathrm{x}}_{k}^{-}=\sum_{j=0}^{p} \alpha_{j}^{m} \mathrm{X}_{k, j}^{\mathrm{x},-}
$$

- $\quad$ Update the CM of the state estimation errors:

$$
\sigma_{\tilde{\mathrm{X}}, k}^{-}=\sum_{j=0}^{p} \alpha_{j}^{c}\left(\mathrm{X}_{k, j}^{\mathrm{X},-}-\hat{\mathrm{x}}_{k}^{-}\right)\left(\mathrm{X}_{k, j}^{\mathrm{X},-}-\hat{\mathrm{X}}_{k}^{-}\right)^{T}
$$

- Calculate the output sigma points matrix at the sampling time $k$ by using the output equation in the model (11):

$$
\mathrm{Y}_{k}=\operatorname{OCV}\left(\hat{z}_{k}, T_{k}\right)+\mathrm{C}_{k} \mathrm{X}_{k, j}^{x,-}+\mathrm{D}_{k} \mathrm{u}_{n, k}+\mathrm{X}_{k-1}^{\xi,+}
$$

- $\quad$ Estimate the output voltage of cells as (19):

$$
\hat{\mathrm{y}}_{\mathrm{k}}=\sum_{j=0}^{p} \alpha_{j}^{m} \mathrm{Y}_{k}
$$

- $\quad$ Update the CM of output voltage estimation errors:

$$
\sigma_{\tilde{\mathrm{y}}, k}^{-}=\sum_{j=0}^{p} \alpha_{j}^{c}\left(\mathrm{Y}_{k}-\hat{\mathrm{y}}_{\mathrm{k}}\right)\left(\mathrm{Y}_{k}-\hat{\mathrm{y}}_{\mathrm{k}}\right)^{T}
$$

- $\quad$ Update the covariance matrices of the state vector estimation error and output voltage estimation error:

$$
\sigma_{\tilde{\mathrm{x}}, \tilde{y}, k}^{-}=\sum_{j=0}^{p} \alpha_{j}^{c}\left(\mathrm{X}_{k, j}^{\mathrm{x},-}-\hat{\mathrm{x}}_{k}^{-}\right)\left(\mathrm{Y}_{k}-\hat{\mathrm{y}}_{\mathrm{k}}\right)^{T}
$$

- $\quad$ Update the estimation gain matrix as (22):

$$
\mathrm{L}_{k}=\frac{\sigma_{\widetilde{\mathrm{x}}, \tilde{y}, k}}{\sigma_{\widetilde{\mathrm{y}}, k}^{-}}
$$

- Update the estimation state vector by using the estimation gain matrix, the measured real output voltages and the estimated output voltage of cells:

$$
\hat{\mathrm{x}}_{k}^{+}=\hat{\mathrm{x}}_{k}^{-}+\mathrm{L}_{k}\left(\mathrm{y}_{k}-\hat{\mathrm{y}}_{\mathrm{k}}\right)
$$

- $\quad$ Update the CM of state estimation error vector:

$$
\sigma_{\tilde{\mathrm{x}}, k}^{+}=\sigma_{\tilde{\mathrm{x}}, k}^{-}-\mathrm{L}_{k} \sigma_{\tilde{\mathrm{y}}, k}^{-} \mathrm{L}_{k}^{T}
$$

\subsection{SoC differences estimation for $\boldsymbol{N}$ cells by second SPKF}

The second SPKF estimates the SoC differences $\Delta \hat{z}_{k}^{(i)}, i=1,2, \ldots, N$, for $N$ cells at the sampling time $k$ as following steps: 
- $\quad$ Form the augmented state vector of cell $i$ at the sampling time $k-1$ by using the SoC difference $\Delta \hat{z}_{k}^{(i)}$ and the mean vector of the SoC difference noises $\bar{w}_{z \Delta}^{(i)}$ :

$$
\Delta \hat{z}_{k-1}^{a,(i)+}=\left[\Delta \hat{z}_{k}^{(i)}, \bar{w}_{z \Delta}^{(i)}\right]
$$

- From the augmented CM of SoC difference estimation error of cell $i$, by using the CM of SoC difference estimation error of cell $i$ at sampling time $k-1$ and the CM of SoC difference noise:

$$
\sigma_{\Delta \widetilde{Z}, k-1}^{a,(i)+}=\operatorname{diag}\left(\sigma_{\Delta \widetilde{z}, k-1}^{(i)+}, \sigma_{w_{\Delta z}^{(i)}}\right)
$$

- Set up the matrix of $p+1$ sigma points of cell $i$ :

$$
\Delta \chi_{k-1}^{a,(i)+}=\left\{\Delta \hat{z}_{k-1}^{a,(i)+}, \Delta \hat{z}_{k-1}^{a,(i)+}+\gamma \sqrt{\sigma_{\Delta \tilde{z}, k-1}^{a,(i)+}}, \Delta \hat{z}_{k-1}^{a,(i)+}-\gamma \sqrt{\sigma_{\Delta \tilde{z}, k-1}^{a,(i)+}} \triangleq\left\{\Delta \chi_{k-1, j}^{\Delta z,(i)+}, \Delta \chi_{k-1, j}^{w_{\Delta z}^{+}}\right\}^{T}\right.
$$

- $\quad$ Calculate the state sigma point matrix of cell $i$ at the sampling time $k$ :

$$
\Delta \chi_{k, j}^{\Delta z,(i)-}=\Delta \chi_{k-1, j}^{\Delta z,(i)+}-\frac{\left(\left(i_{n, k}-\hat{\imath}_{b, k}\right)+\Delta \chi_{k-1, y}^{w_{\Delta z}^{+}}\right)}{3600 Q} \quad j=0,1,2, \ldots, p
$$

- $\quad$ Estimate the SoC difference of cell $i$ at the sampling time:

$$
\Delta \hat{z}_{k}^{(i)-}=\sum_{j=0}^{p} \alpha_{j}^{m} \Delta \chi_{k, j}^{\Delta z,(i)}
$$

- Update the CM of SoC difference estimation error of cell:

$$
\sigma_{\Delta \tilde{z}, k}^{(i)-}=\sum_{j=0}^{p} \alpha_{j}^{c}\left(\Delta \chi_{k, j}^{\Delta z,(i)-}-\Delta \hat{z}_{k}^{(i)-}\right)\left(\Delta \chi_{k, j}^{\Delta z,(i)-}-\Delta \hat{z}_{k}^{(i)-}\right)^{T}
$$

- Calculate the output sigma points matrix of cell $i$ :

$$
\begin{aligned}
& \mathrm{Y}_{k}^{(i)}=\operatorname{OCV}\left(\bar{z}_{k}+\Delta \hat{z}_{k}^{(i)}, T_{k}\right)-i_{n, k} \Delta R^{(i)}+ \\
& M^{(i)} \hat{h}_{k}-R_{1}^{(i)} C_{1}^{(i)} \hat{\imath}_{R, 1}-R_{2}^{(i)} C_{2}^{(i)} \hat{\imath}_{R, 2}-R_{0}\left(i_{n, k}-\hat{\imath}_{b}^{k}\right)
\end{aligned}
$$

- $\quad$ Estimate the output voltage of cell $i$ :

$$
\hat{y}_{k}^{(i)}=\sum_{j=0}^{p} \alpha_{j}^{m} \mathrm{Y}_{k}^{(i)}
$$

- $\quad$ Update the CM of output voltage estimation error of cell $i$ :

$$
\sigma_{\widetilde{y}, k}^{(i)}=\sum_{j=0}^{p} \alpha_{j}^{c}\left(\mathrm{Y}_{k}^{(i)}-\hat{y}_{k}^{(i)}\right)\left(\mathrm{Y}_{k}^{(i)}-\hat{y}_{k}^{(i)}\right)^{T}
$$

- $\quad$ Update the CM of SoC difference estimation error and output voltage estimation error of cell $i$ :

$$
\sigma_{\Delta \tilde{z}, \tilde{y}, k}^{(i)-}=\sum_{j=0}^{p} \alpha_{j}^{c}\left(\Delta \chi_{k, j}^{\Delta z,(i)-}-\Delta \hat{z}_{k}^{(i)-}\right)\left(\mathrm{Y}_{k}^{(i)}-\hat{y}_{k}^{(i)}\right)^{T}
$$

- $\quad$ Update the state estimation matrix of cell:

$$
L_{k}^{(i)}=\frac{\sigma_{\Delta \tilde{z}, \tilde{y}, k}^{(i)-}}{\sigma_{\tilde{y}, k}^{(i)}}
$$

- $\quad$ Estimate the SoC difference of: 


$$
\Delta \hat{z}_{k}^{(i)+}=\Delta \hat{z}_{k}^{(i)-}+L_{k}^{(i)}\left(y_{n, k}^{(i)}-\hat{y}_{k}^{(i)}\right)
$$

- $\quad$ Update the CM of SoC difference estimation error of cell $i$ :

$$
\sigma_{\Delta \tilde{z}, k}^{(i)+}=\sigma_{\Delta \widetilde{z}, k}^{(i)-}-L_{k}^{(i)} \sigma_{\widetilde{y}, k}^{(i)} L_{k}^{(i)}
$$

\subsection{SoC estimation of cells}

The SoC of cell $i$ calculated as (38):

$$
\hat{z}_{k}^{(i)+}=\hat{z}_{k}^{+}+\Delta \hat{z}_{k}^{(i)+}
$$

\section{RESULTS AND DISCUSSION}

The experimental system of SoC estimation is described in Figure 6, the components in the experimental system are listed as: i) The $\mathrm{LiB}$ samsung is formed by 7 cells connected in series with parameters listed in Table 1; measurement modul is created by 7 voltage difference measurement circuits, 1 current measurement curcuit, 1 temperature sensor. The voltage difference signals are to the digital signals 10 bit and transferred to the personal computer (PC) by RS485; ii) the PC implements the SoC estimation program for cells, the configuration of PC is core (TM) i3-6100 CPU@3.70 GHZ, 4 cores, RAM 8 GB; the MATLAB software is used to implement the SPKF algorithms, the LabVIEW 2018 [22] is used to make the graphic user interface, the MATLAB software is embedded to the LabVIEW to run the SoC estimation; iii) the load is motor BLDC, YONG-d67 $240 \mathrm{~W}, 24 \mathrm{~V}, 10 \mathrm{~A}$, the maximum speed is $295 \mathrm{rmp}$; iv) the charge source is owon, $195 \mathrm{~W}$ with 2 programmable channel $36 \mathrm{~V}$, maximum current $6 \mathrm{~A}$; and v) the SoC estimation algorithms are coded in MATLAB script, then embedded to the LabVIEW environment. The sampling time interval is 1 second. The algorithm flowchart of SoC estimation for $N$ cell in shown in Figure 7.

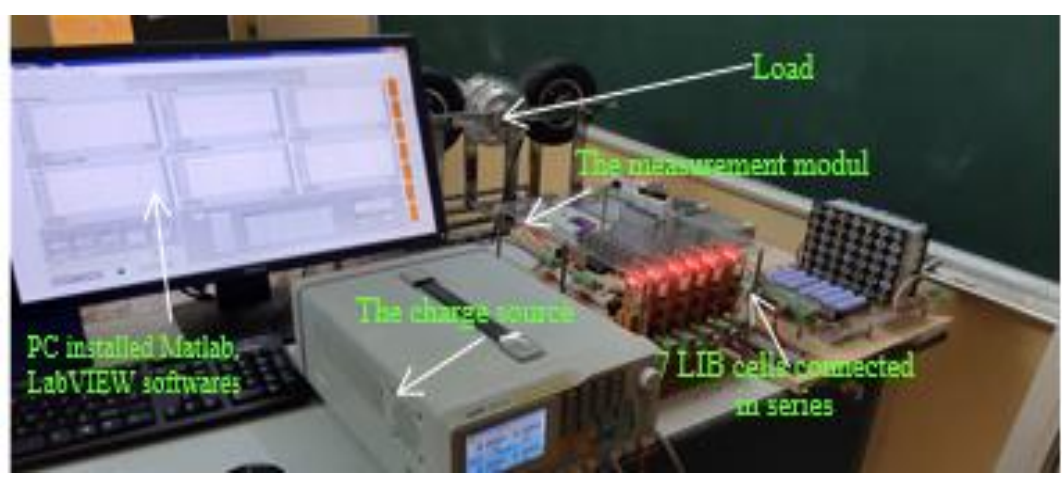

Figure 6. The experimental system of SoC estimation

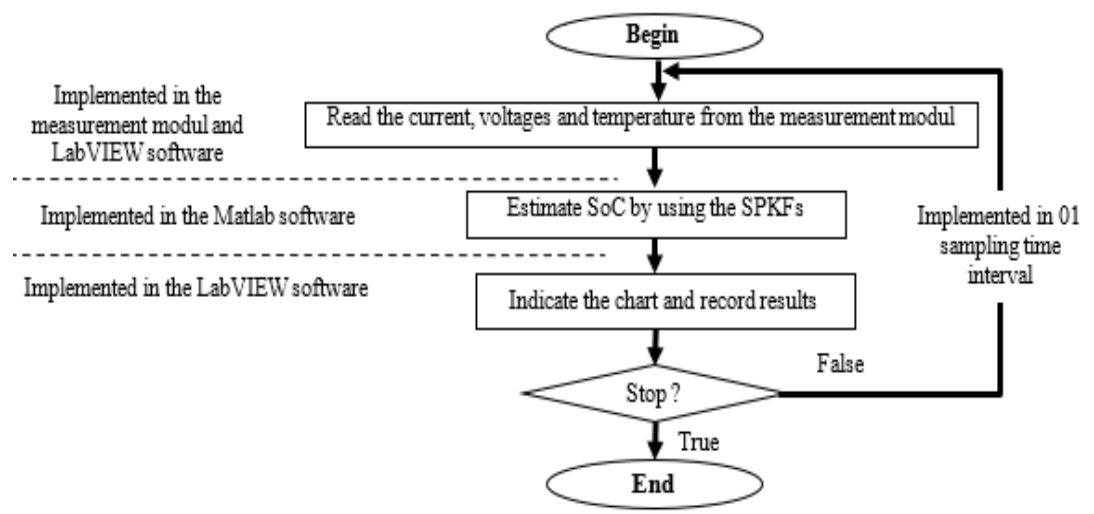

Figure 7. The algorithm flowchart of SoC estimation 


\subsection{The SoC estimation results for 1 cell}

In this part, the SoC estimation results for 1 cell is presented, the Figure 8(a) describes the current, voltage and temperature of the cell. The noise distributions of measured currrent, voltage and temperature are plotted in Figure 8(b), the Figure 8(c) and the Figure 8(d). The current noise was distributed inside from $-0.8 A$ to $0.8 A$ caused by shunt-resistor with the sensitivity $15 \mathrm{mV} / A$. The range of the voltage noise is range of $-0.03 \mathrm{~V}$ to $0.03 \mathrm{~V}$, the distribution of temperature noise is in the range from $-0.7{ }^{0} \mathrm{C}$ to $0.7{ }^{0} \mathrm{C}$. Figure 9 shows the results obtained when performing the SoC estimation algorithm corresponding to current, temperature and voltage inputs as shown in Figure 8 using the second-order model of cell with the parameters described in Table 1.

The Figure 9(a) describes the comparison between real cell voltage and estimated voltage calculated by cell model, the voltage error is plotted in Figure 9(b) with $0.1 \mathrm{~V}$ and $-0.075 \mathrm{~V}$ of maximum for discharge and charge, respectively. This maximum error is caused when there is the changing of charge to discharge vice versa. The cause of voltage error is the dependence of model on the temperature varying in the test, the parameters of cell were determined at particular temperatures $-5{ }^{0} \mathrm{C}, 0{ }^{0} \mathrm{C}, 5{ }^{0} \mathrm{C}, 10{ }^{0} \mathrm{C}, 15^{0} \mathrm{C}, 20^{\circ} \mathrm{C}$, $25{ }^{0} \mathrm{C}, 30{ }^{\circ} \mathrm{C}, 35^{0} \mathrm{C}$. In the test, the temperature of cell raises from $25 .{ }^{0} \mathrm{C}$ at to $31.5{ }^{0} \mathrm{C}$ as plotted in Figure 9(c). The Figure 9(c) also plotted the estimated SoC of cell. After 1 hour with the discharging $(3 A)$ and the charging $(1.5 A)$ alternately, The SoC of cell tends to decrease from $92.5 \%$ at beginning to $65 \%$ at the end of the test. The SoC estimation error is shown in Figure 9(d) by comparing to criteria $\hat{z}_{k} \pm 3 \sigma$.

The criteria $3 \sigma$ decreases gradually from the beginning of the test to the end of the test as plotted in Figure 9(e), we can see that the accuracy of SoC estimation of the cell described by the criteria $3 \sigma$ at the end of test was $0.0125 \%$. The Figure 9 (f) plots the updating of matrix $\sigma_{\tilde{x}}^{+}$by the time. At the times, there are the changings of charge to discharge vice versa, the voltage errors are large, some elements of matrix $\sigma_{\tilde{x}}^{+}$need to be changed in order to keep estimating SoC smoothly.

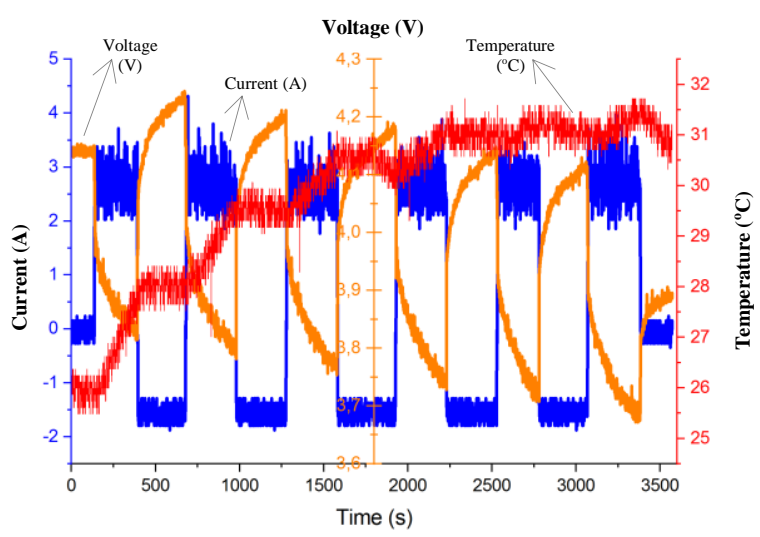

(a)

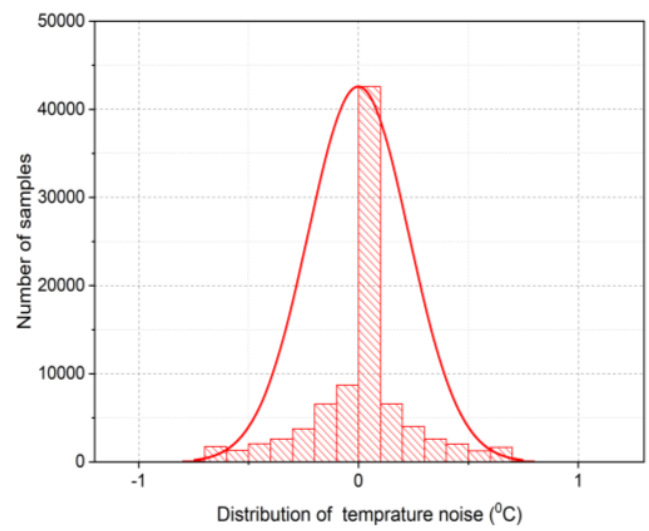

(c)

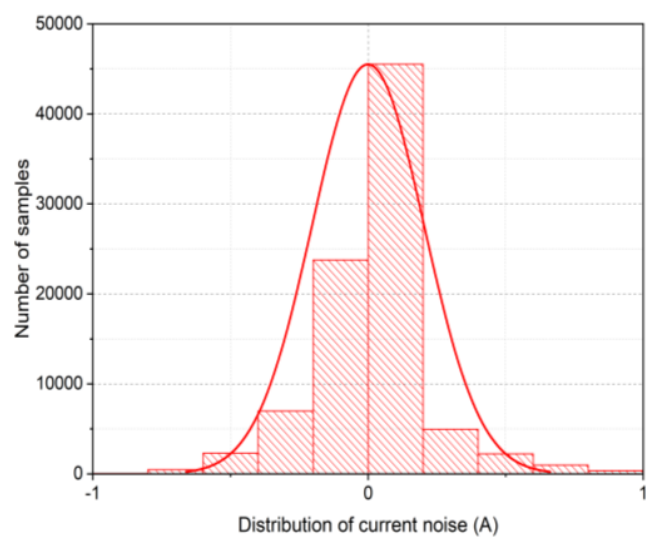

(b)

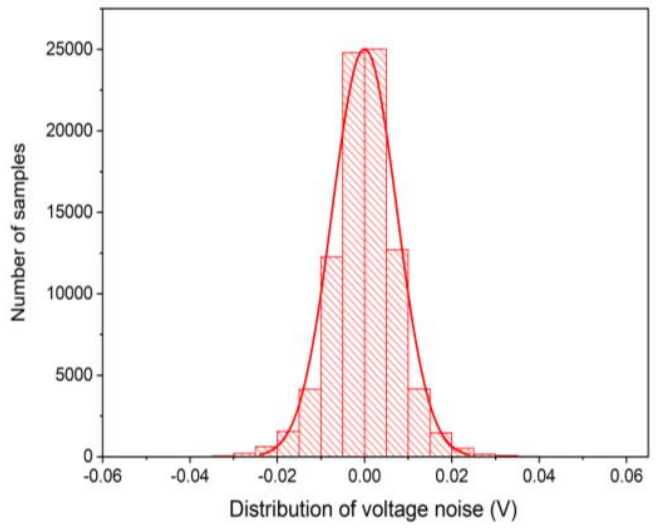

(d)

Figure 8. The measurements and distributions, (a) the current, voltage and temperature of cell in the test,

(b) the noise distributions of current, (c) temperature, and (d) voltage 


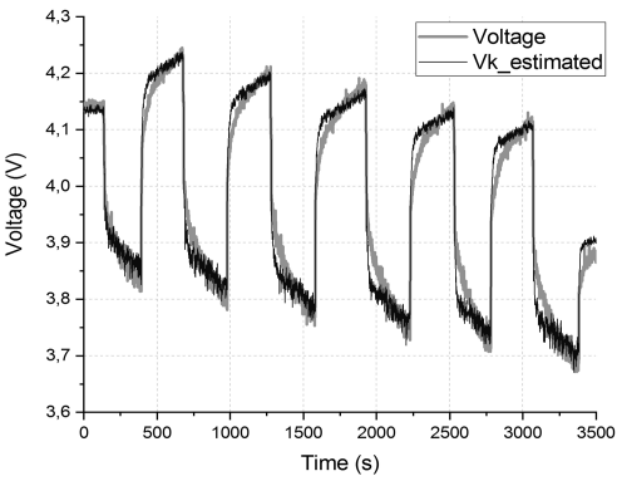

(a)

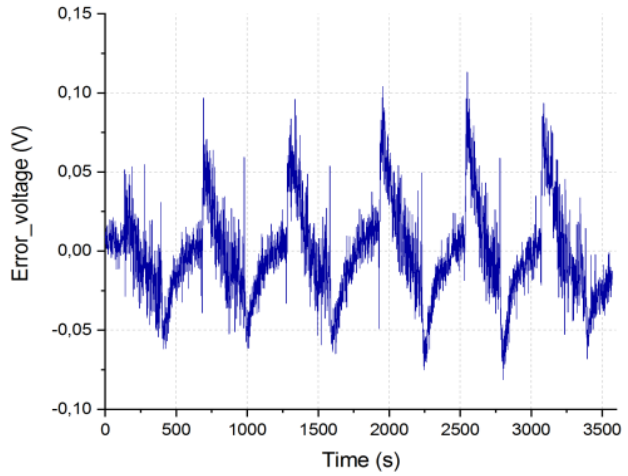

(b)

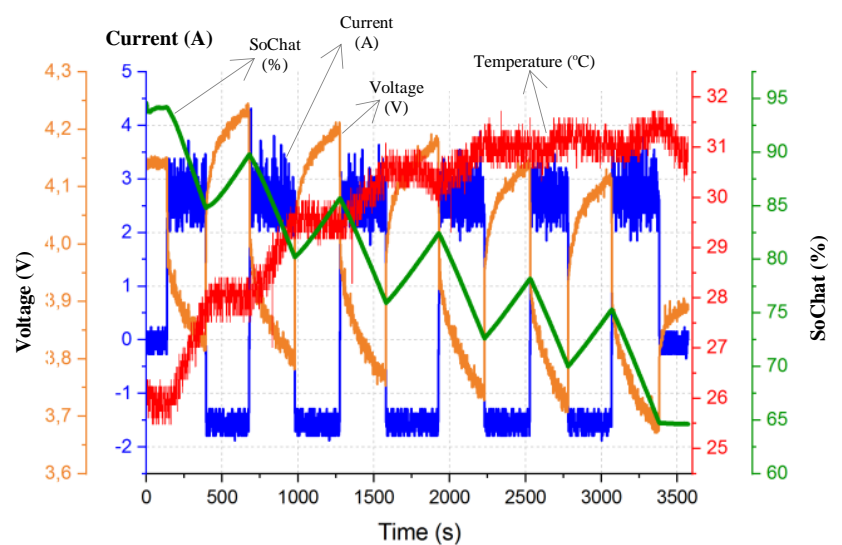

(c)

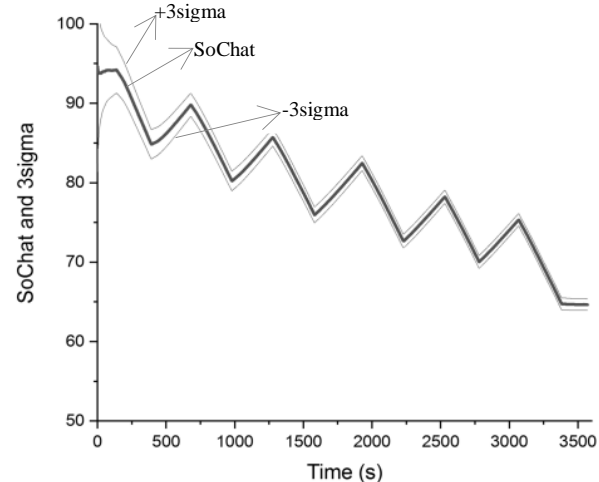

(d)

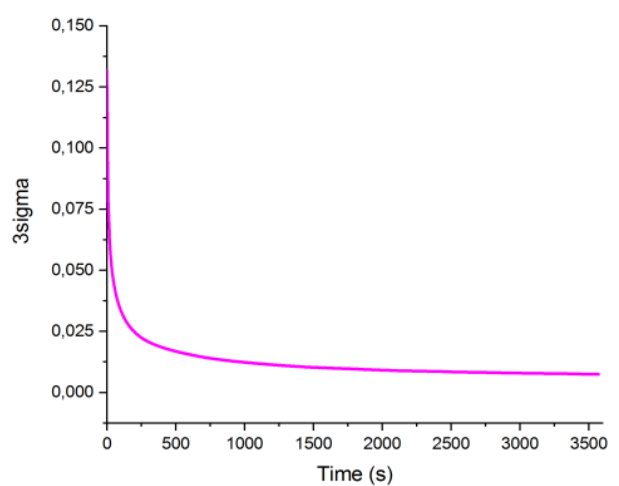

(e)

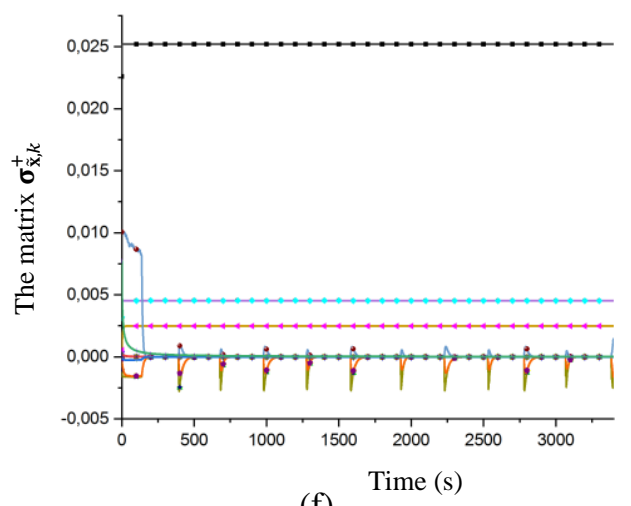

(f)

Figure 9. The SoC estimation results for 1 cell, (a) the voltage and estimated voltage, (b) voltage error, (c) the estimated SoC and temperature, (d) estimated $\mathrm{SoC} \pm 3 \sigma$, (e) criteria $3 \sigma$, and (f) the matrix $\boldsymbol{\sigma}_{\tilde{\mathbf{x}}, k}^{+}$ 


\subsection{The SoC estimation results for 7 cells}

In this part, the SoC estimation results for 7 cells connected in series are presented. Suppose that all cells are the same with parameters listed in Table 1. The Figure 10(a) plots the cells current, the Figure 10(b) and Figure 10(c) depicts the voltages of cells and temperature, respectively, the distributions of noises in current, voltages and temperature are plotted in Figures 10(d), 10(e) and 10(f). The estimated SoC for 7 cells is presented in Figure 11 and Figure 12 (in appendix), in which the Figure 11(a) is the estimated average SoC for 7 cells, the Figure 11(b) plots the estimated SoC differences of cells.

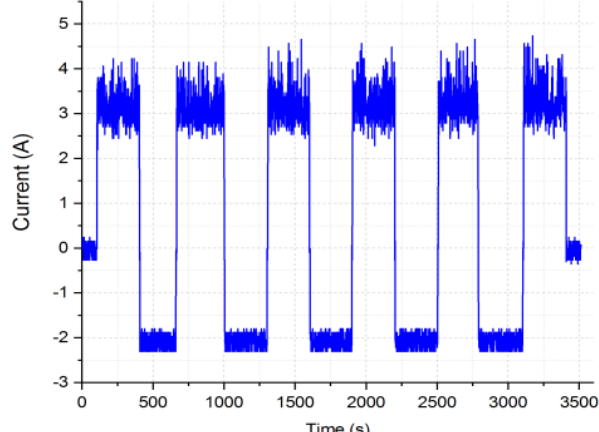

(a)

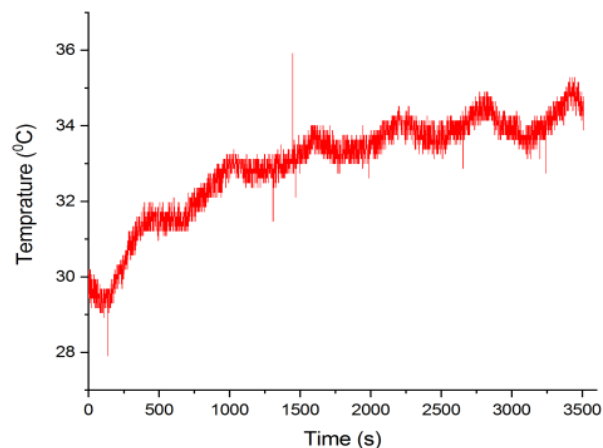

(c)

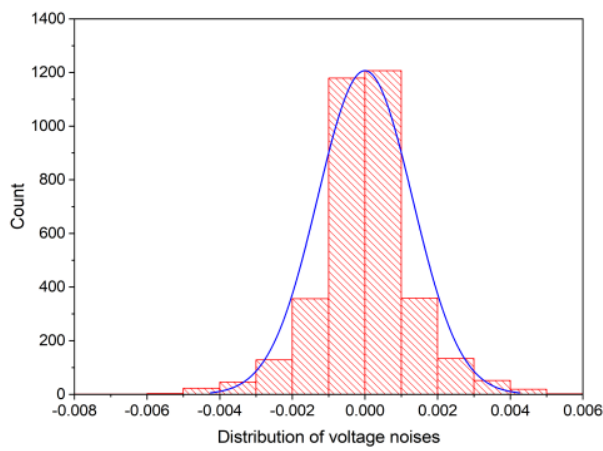

(e)

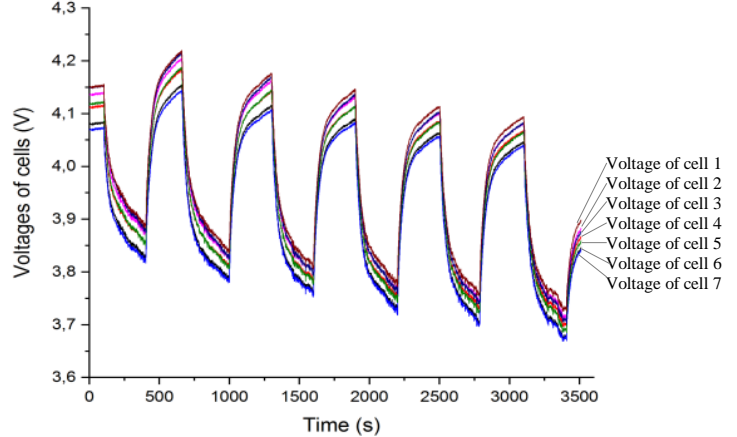

(b)

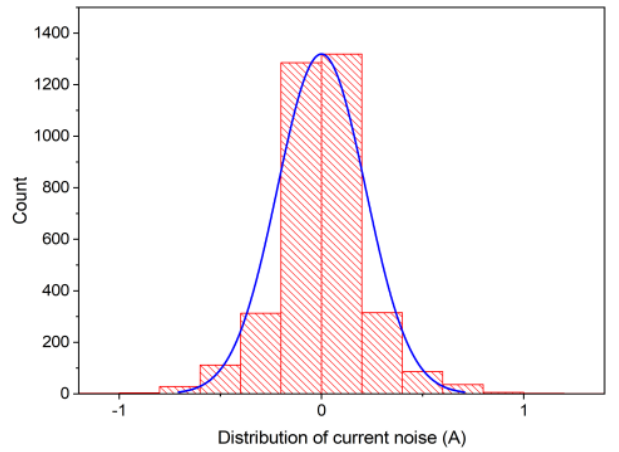

(d)

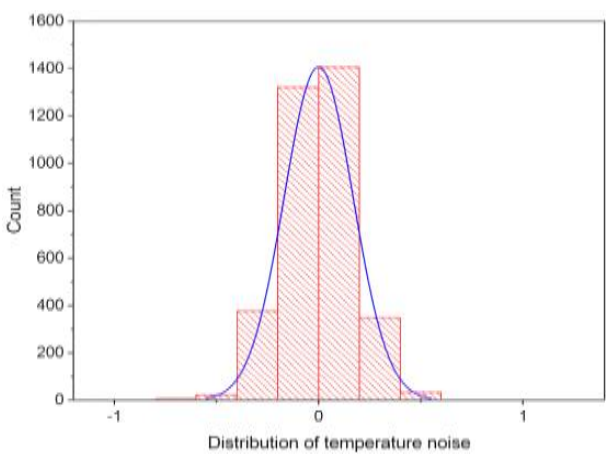

(f)

Figure 10. The measurements and distributions, (a) the current of 7 cells, (b) the voltages of cells, (c) the temperature of cell, (d) the distributions of noise in current, (e) voltages and (f) temperature are plotted

The Figure 12 plots the estimated $\mathrm{SoC}$ of each cell and comparing with criteria $3 \sigma$, in which: Figure 12(a) and Figure 12(b) are the estimated SoC and comparing with criteria $3 \sigma$ for cell 1, similarity for the cells from 2 to 7 are plotted in Figure 12(c) to Figure 12(o). The Figure 13(a) shows the varying of matrix $\sigma_{\tilde{z}}^{+}$in the test, the Figure 13(b) plots the varying of criteria $3 \sigma$. The mean of the criteria $3 \sigma$ for cells at the end of the test is approximately $0.5 \%$. Figures 12 and 13 see in appendix. 


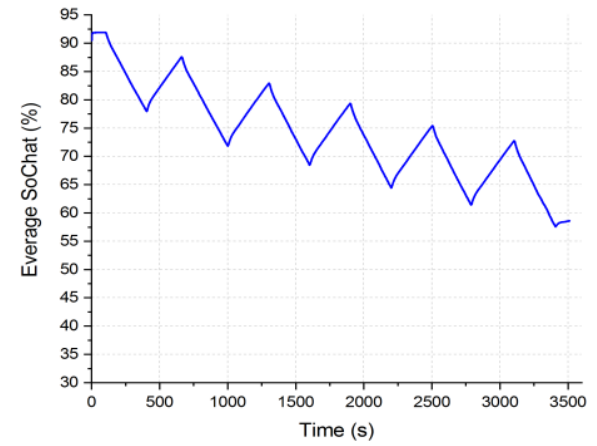

(a)

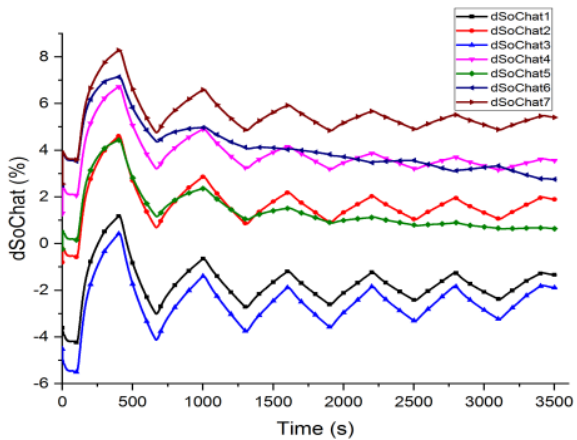

(b)

Figure 11. The estimated SoC for 7 cells, (a) the estimated average SoC, and (b) the estimated SoC difference of cells

\section{CONCLUSION}

This paper deals with the method for SoC estimation of LIB with cells connected in series. In this works, the cell's model is represented by the second-order equivalent circuit model taking into account the measurement disturbances and the current sensor bias. Using 02 SPKF filters to estimate the average SoC of cells, the current sensor bias and the SoC differences, the estimated SoC of each cell is is calculated by sum of pack's everage SoC estimated by first SPKF and SoC differences estimated by second SPKF. This allows reducing the SoC estimation times to 2 times, this is a very effective solution when the battery pack has a large number of cells. The practical test is applied to 7 samsung ICR18650-22P lithium-Ion battery cells with the discharging $(3 \mathrm{~A})$ and the charging $(1.5 \mathrm{~A})$ alternately in 1 hour. The estimated SoC is quite accuracy with the criteria $3 \sigma$ of estimated SoC error is approximately 0.5. In this work, we suppose that all cells are physically identical, the equivalent circuit model with parameters listed in Table 1 are linearized at some particular temperatures and applied for all cells. This is clear evidence that those factors contribute to the SoC and current sensor bias estimation error observed in the test. In future work, the model of each cell to be updated adaptively by temperature will be researched.

\section{APPENDIX}

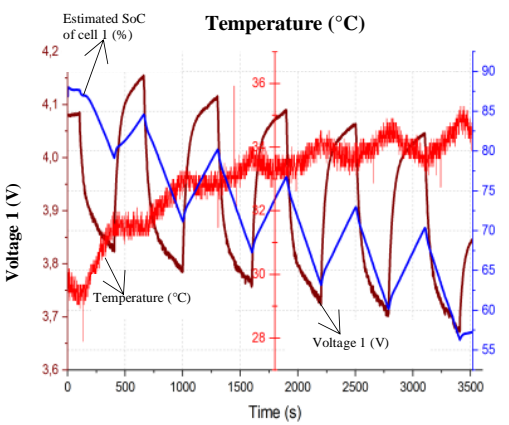

(a)

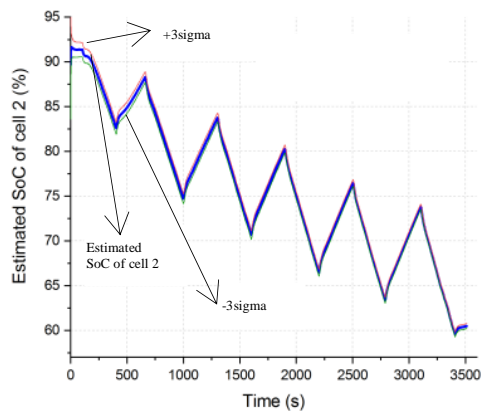

(d)

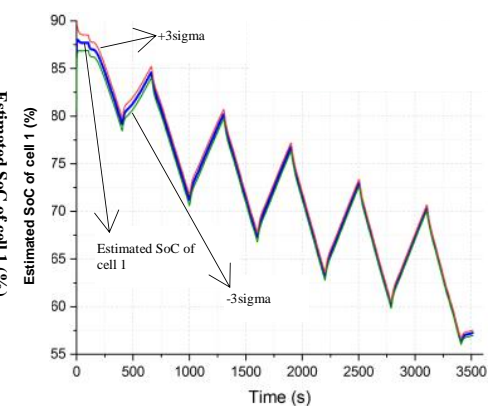

(b)

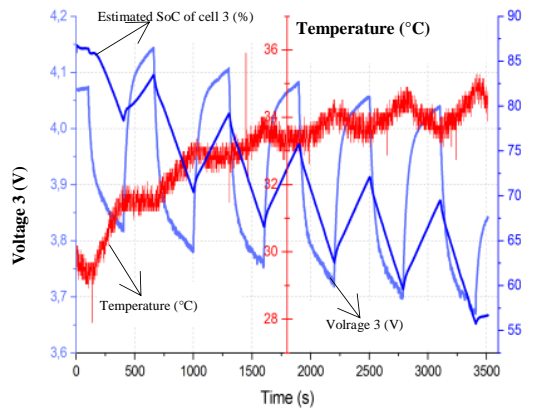

(e)

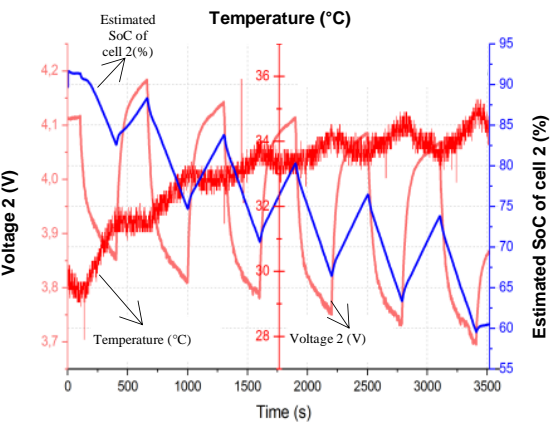

(c)

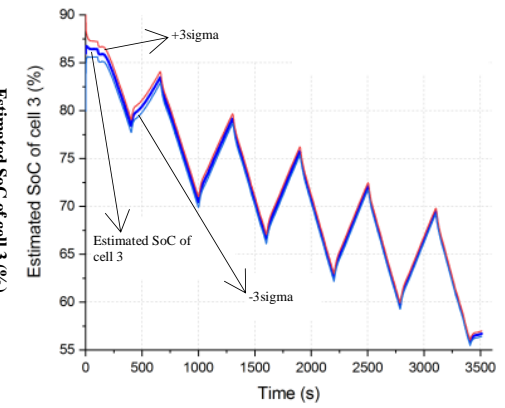

(f) 


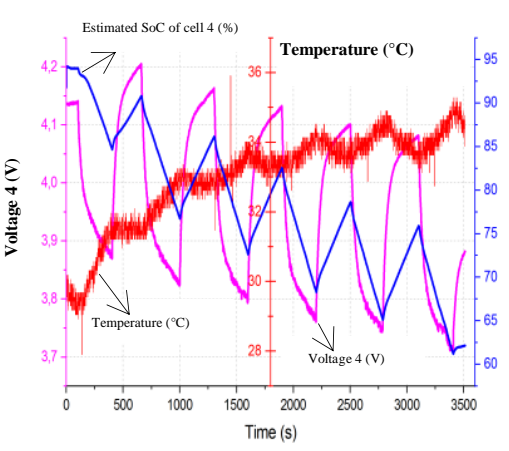

$(\mathrm{g})$

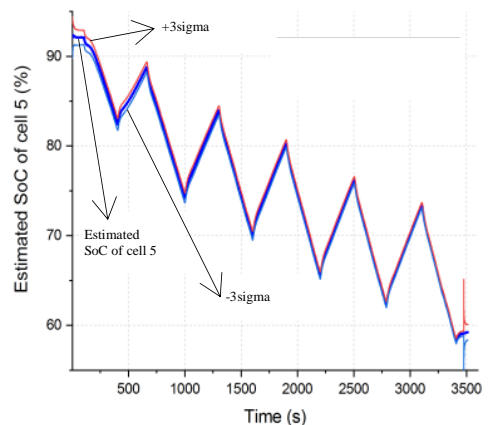

(j)

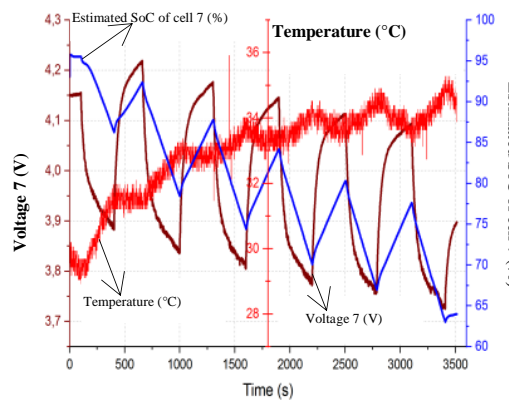

(m)

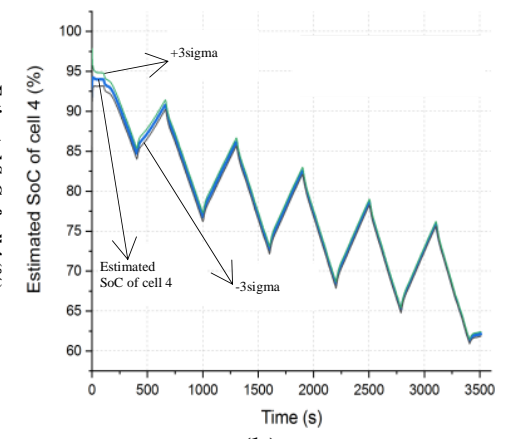

(h)

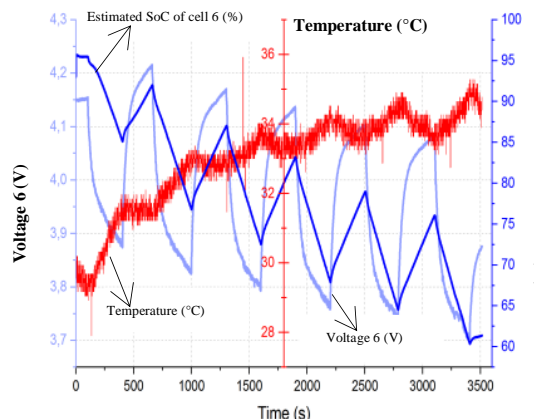

(k)

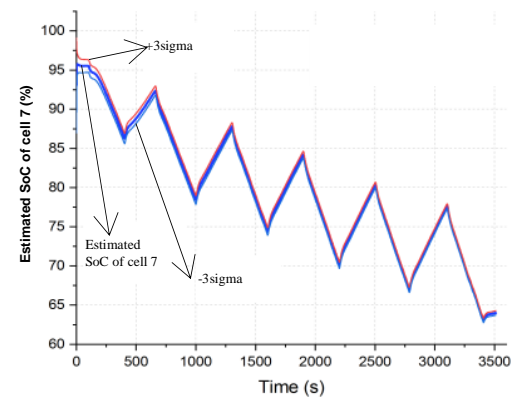

(n)

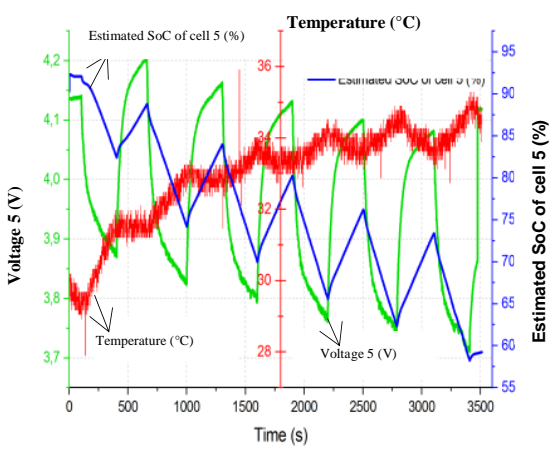

(i)

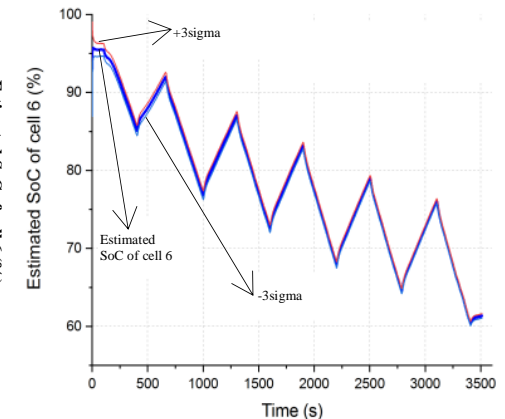

(1)

Figure 12. The estimated SoC for each cell and the comparing with criteria $3 \sigma$, (a) the measurements for cell 1, (b) estimated SoC for cell 1, (c) the measurements for cell 2, (d) estimated SoC for cell 2, (e) the measurements for cell 3, and (f) estimated SoC for cell 3, (g) the measurements for cell 4, (h) estimated SoC for cell 4, (i) the measurements for cell 5, (j) estimated SoC for cell 5, (k) the measurements for cell 6, (l) estimated SoC for cell 6, (m) the measurements for cell 7, and (n) estimated SoC for cell 7

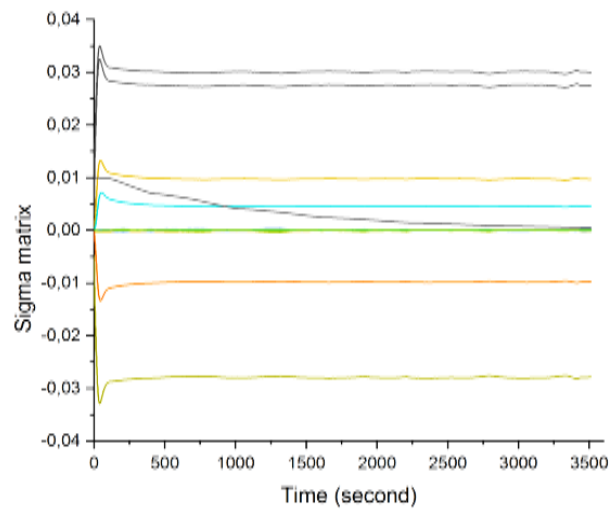

(a)

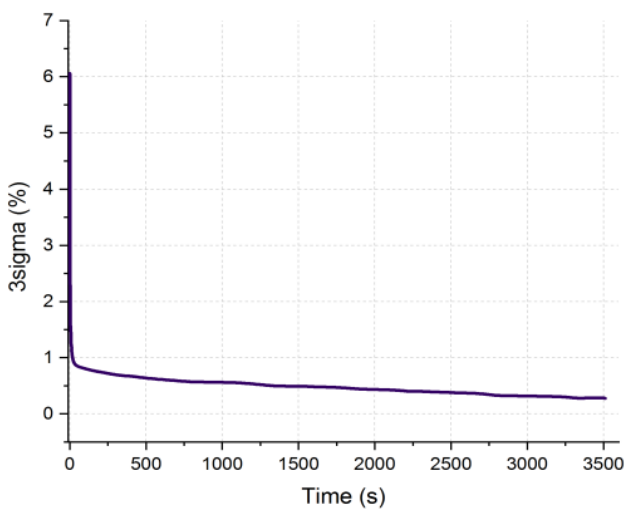

(b)

Figure 13. The matrix $\sigma_{\tilde{\mathrm{z}}}^{+}$and criteria $3 \sigma$, (a) the varying of the matrix $\sigma_{\tilde{\mathrm{z}}}^{+}$in the test and (b) criteria $3 \sigma$ 


\section{ACKNOWLEDGEMENTS}

This research was supported by Thai Nguyen University of Technology, TNUT, Vietnam.

\section{REFERENCES}

[1] J. Warner, "Lithium-ion battery packs for EVs," in Lithium-Ion Batteries, Elsevier, 2014, pp. 127-150.

[2] K. M. Venkatachalam and V. Saravanan, "Performance evaluation and load demand management of grid connected hybrid windsolar-battery system," International Journal of Applied Power Engineering (IJAPE), vol. 9, no. 3, pp. 223-244, Dec. 2020, doi: 10.11591/ijape.v9.i3.pp223-244.

[3] X. Lin, "Theoretical analysis of battery SOC estimation errors under sensor bias and variance," IEEE Transactions on Industrial Electronics, vol. 65, no. 9, pp. 7138-7148, Sep. 2018, doi: 10.1109/TIE.2018.2795521.

[4] C. Yuan, Y. Deng, T. Li, and F. Yang, "Manufacturing energy analysis of lithium ion battery pack for electric vehicles," CIRP Annals, vol. 66, no. 1, pp. 53-56, 2017, doi: 10.1016/j.cirp.2017.04.109.

[5] D. N. T. How, M. A. Hannan, M. S. Hossain Lipu, and P. J. Ker, "State of charge estimation for lithium-ion batteries using model-based and data-driven methods: a review," IEEE Access, vol. 7, pp. 136116-136136, 2019, doi: 10.1109/ACCESS.2019.2942213.

[6] Y. Zhou and X. Li, "Overview of lithium-ion battery SOC estimation," in 2015 IEEE International Conference on Information and Automation, Aug. 2015, pp. 2454-2459, doi: 10.1109/ICInfA.2015.7279698.

[7] J. Rivera-Barrera, N. Muñoz-Galeano, and H. Sarmiento-Maldonado, "SoC estimation for lithium-ion batteries: review and future challenges," Electronics, vol. 6, no. 4, p. 102, Nov. 2017, doi: 10.3390/electronics6040102.

[8] J. Xie, J. Ma, and K. Bai, "Enhanced coulomb counting method for state-of-charge estimation of lithium-ion batteries based on peukert's law and coulombic efficiency," Journal of Power Electronics, vol. 18, no. 3, pp. 910-922, 2018.

[9] P. Manoharan, M. R, K. K, and S. R, "SoC estimation and monitoring of li-ion cell using Kalman-Filter Algorithm," Indonesian Journal of Electrical Engineering and Informatics (IJEEI), vol. 6, no. 4, Dec. 2018, doi: 10.11591/ijeei.v6i4.548.

[10] M. Souaihia, B. Belmadani, and R. Taleb, "A robust state of charge estimation for multiple models of lead acid battery using adaptive extended Kalman filter," Bulletin of Electrical Engineering and Informatics (BEEI), vol. 9, no. 1, pp. 1-11, Feb. 2020, doi: 10.11591/eei.v9i1.1486.

[11] Z. L. Huan, T. Tomohiro, and A. Tofael, "Leader-follower tracking system for agricultural vehicles: Fusion of laser and odometry positioning using extended kalman filter," IAES International Journal of Robotics and Automation (IJRA), vol. 4, no. 1, pp. 1-18, Mar. 2015, doi: 10.11591/ijra.v4i1.pp1-18.

[12] V. Sangwan, R. Kumar, and A. K. Rathore, "State-of-charge estimation for li-ion battery using extended Kalman filter (EKF) and central difference Kalman filter (CDKF)," in 2017 IEEE Industry Applications Society Annual Meeting, Oct. 2017, pp. 1-6, doi: 10.1109/IAS.2017.8101722.

[13] N. H. Mohd Amin, M. R. Ab Ghani, A. Jidin, Z. Jano, and T. Sutikno, "A review of hybrid battery management system (H-BMS) for EV," TELKOMNIKA (Telecommunication Computing Electronics and Control), vol. 16, no. 3, pp. 1004-1012, Jun. 2018, doi: 10.12928/telkomnika.v16i3.8743.

[14] W. Zheng, B. Xia, W. Wang, Y. Lai, M. Wang, and H. Wang, "State of charge estimation for power lithium-ion battery using a fuzzy logic sliding mode observer," Energies, vol. 12, no. 13, p. 2491, Jun. 2019, doi: 10.3390/en12132491.

[15] M. Charkhgard and M. Farrokhi, "State-of-charge estimation for lithium-ion batteries using neural networks and EKF," IEEE Transactions on Industrial Electronics, vol. 57, no. 12, pp. 4178-4187, Dec. 2010, doi: 10.1109/TIE.2010.2043035.

[16] F. Sun, R. Xiong, and H. He, "A systematic state-of-charge estimation framework for multi-cell battery pack in electric vehicles using bias correction technique," Applied Energy, vol. 162, pp. 1399-1409, Jan. 2016, doi: 10.1016/j.apenergy.2014.12.021.

[17] N.-T. Tran, A. Khan, T.-T. Nguyen, D.-W. Kim, and W. Choi, "SOC estimation of multiple lithium-ion battery cells in a module using a nonlinear state observer and online parameter estimation," Energies, vol. 11, no. 7, Jun. 2018, doi: 10.3390/en11071620.

[18] D. Saji, P. S. Babu, and K. Ilango, "SoC estimation of lithium ion battery using combined coulomb counting and fuzzy logic method," in 2019 4th International Conference on Recent Trends on Electronics, Information, Communication \& Technology (RTEICT), May 2019, pp. 948-952, doi: 10.1109/RTEICT46194.2019.9016956.

[19] A. M. Alsabari, M. K. Hassan, A. CS, and R. Zafira, "Modeling and validation of lithium-ion battery with initial state of charge estimation," Indonesian Journal of Electrical Engineering and Computer Science (IJEECS), vol. 21, no. 3, pp. 1317-1331, Mar. 2021, doi: 10.11591/ijeecs.v21.i3.pp1317-1331.

[20] S. Soeprapto, R. N. Hasanah, and T. Taufik, "Battery management system on electric bike using Lithium-Ion 18650," International Journal of Power Electronics and Drive Systems (IJPEDS), vol. 10, no. 3, pp. 1529-1537, Sep. 2019, doi: 10.11591/ijpeds.v10.i3.pp1529-1537.

[21] W. Fan and Y. Li, "Accuracy analysis of sigma-point Kalman filters," in 2009 Chinese Control and Decision Conference, Jun. 2009, pp. 2883-2888, doi: 10.1109/CCDC.2009.5192691.

[22] B. N. Kumar Reddy, N. Suresh, and J. V. N. Ramesh, "A gracefully degrading and energy-efficient FPGA programming using LabVIEW," International Journal of Reconfigurable and Embedded Systems (IJRES), vol. 5, no. 3, pp. 165-175, Nov. 2016, doi: 10.11591/ijres.v5.i3.pp165-175.

[23] Qin Wang, C. Rizos, Yong Li, and Shiyi Li, “Application of a sigma-point Kalman filter for alignment of MEMS-IMU," in 2008 IEEE/ION Position, Location and Navigation Symposium, 2008, pp. 44-52, doi: 10.1109/PLANS.2008.4569968.

[24] C. Nguyen Van, "State estimation based on sigma point kalman filter for suspension system in presence of road excitation influenced by velocity of the car," Journal of Control Science and Engineering, vol. 2019, pp. 1-16, Nov. 2019, doi: $10.1155 / 2019 / 6898756$

[25] C. Nguyen Van and T. Nguyen Vinh, "SOC estimation of the lithium-ion battery pack using a sigma point kalman filter based on a cell's second order dynamic model," Applied Sciences, vol. 10, no. 5, Mar. 2020, doi: 10.3390/app10051896.

[26] O. Bayasli and H. Salhi, "The cubic root unscented kalman filter to estimate the position and orientation of mobile robot trajectory," International Journal of Electrical and Computer Engineering (IJECE), vol. 10, no. 5, pp. 5243-5250, Oct. 2020, doi: 10.11591/ijece.v10i5.pp5243-5250.

[27] V. D. Merwe and R. Sigma, "Point Kalman filters for probabilistic inference in dynamic state-space models," Oregon Health and Science University, 2004. 


\section{BIOGRAPHIES OF AUTHORS}

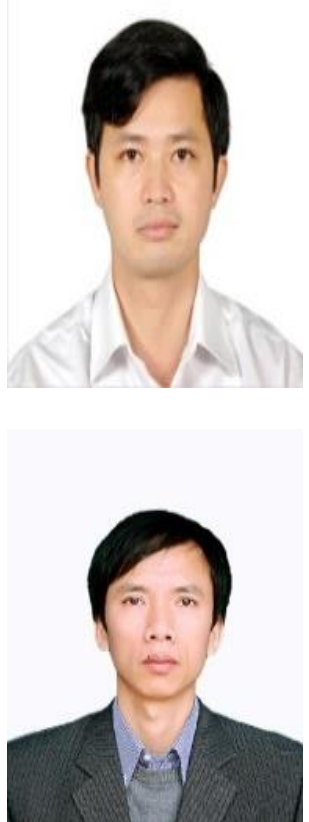

Chi Nguyen Van (D) $8 \mathrm{SC}$ P received the PhD degree in Instrument and Control Engineering from Hanoi University of Science and Technology (HUST) in 2012. He is currently working as a Associate Professor with Institute of High-Technology Research and Development for Industry (RIAT), Thai Nguyen University of Technology (TNUT), Viet Nam. His research interests include nonlinear control, adaptive control, optimal control and system identification for Lithium Ion Batterry. He can be contacted at email: ngchi@tnut.edu.vn

Thuy Nguyen Vinh (iD SC P received the MSs degree in Automation Engineering from Thai Nguyen University of Technology (TNUT) in 2005. He is currently working as a researcher with Institute of High-Technology Research and Development for Industry (RIAT), Thai Nguyen University of Technology (TNUT) His research interests optimal control and system identification for Lithium Ion Batterry. He can be contacted at email: nguyenvinhthuytdh@ tnut.edu.vn 